\title{
Competitive Exclusion in a Discrete Stage-Structured Two Species Model
}

\author{
A. S. Ackleh* and P. Zhang \\ Department of Mathematics, University of Louisiana at Lafayette \\ Lafayette, Louisiana 70504
}

\begin{abstract}
We develop a stage-structured model that describes the dynamics of two competing species each of which have sexual and clonal reproduction. This is typical of many plants including irises. We first analyze the dynamical behavior of a single species model. We show that when the inherent net reproductive number is smaller than one then the population will go to extinction and if it is larger than one then an interior equilibrium exists and it is globally asymptotically stable. Then we analyze the two-species model and establish conditions on the reproduction and survivorship rates that lead to competitive exclusion. We show that the winner species is the one that attains higher density at which its net reproductive number equals unity. Numerical results corroborating the theoretical ones are also presented.
\end{abstract}

Key words: stage-structured model, net reproductive number, competitive exclusion AMS subject classification: 92D25

\section{Introduction}

Competition among species is very common. It can be considered as a relationship between organisms or species, in which members of individuals are adversely affected by the presence of those requiring the same living resources. For example, two plants growing close to each other compete for the same resources such as light, space, water and nutrients. If the environmental resources are not sufficient, then both plants will not perform as well as when they are growing alone. If the competing members are of the same species, then the interaction between them is known as

${ }^{*}$ Corresponding author. E-mail:ackleh@louisiana.edu 
intraspecific competition. While if competing members are of different species then such interaction is called interspecific competition. The Louisiana blue flag iris (I. hexagona) [14] and the cultivated yellow flag iris (I. pseudacorus) [11] living in the Louisiana Gulf Coast provide such an example. These two iris species have similar life history and are ecologically closely related. Both of them reproduce clonally and sexually and endure a wide range of environmental conditions [10]. However, some recent research $[10,12,13]$ shows that they respond differently to salinity stress.

In [10], the authors develop a quantitative stage-structured model as a tool to investigate the dynamics of these two competing iris populations. By using empirical data for model parameters and applying Monte Carlo simulations, the authors show that competitive exclusion occurs between these two species, where yellow iris excludes the blue iris in about $82 \%$ of encounters in freshwater habitats. While the blue iris excludes the yellow iris in $99 \%$ of encounters in brackish marsh [10].

The purpose of this paper is to investigate theoretically the dynamics of two ecologically similar species under competition, such as the blue and yellow irises, and to understand the conditions under which competitive exclusion between these populations occurs. To this end, in Section 2 , we develop and analyze a stage-structured one-species model which describes the dynamics of one population with intraspecific competition. Then in Section 3, we present a two-species model which describes the dynamics of two populations with interspecific competition and provide conditions on the net reproductive number that leads to competitive exclusion. In Section 4, we provide numerical results and in Section 5 we present a few concluding remarks. Finally, in the Appendix we recall two results that are needed to establish the global stability in this paper.

\section{Three-Stage Single-Species Model}

We assume that the population is divided into three stages: seeds, juveniles (seedlings), and adults (flowering plants) which produce seeds via sexual reproduction and juveniles via clonal reproduction. We denote by $x_{t}, y_{t}, z_{t}$ the number of seeds, juveniles and adults, respectively, at time $t$. Let $s_{1}$ represent the survivorship of the seeds which is assumed to be constant. Let $s_{2}\left(y_{t}+z_{t}\right)$ represent the survivorship of the juveniles at time $t$, and $s_{3}\left(y_{t}+z_{t}\right)$ represent the survivorship of the adults at time $t$ which are assumed to be density dependent due to competition for resources between juveniles and adults. We denote by $b_{1}$ the number of seeds produced by adults per time step (sexual reproduction rate) and by $b_{2}$ the number of juveniles (seedlings) formed by adults per time step (clonal reproduction rate). We assume that the time unit is chosen such that the seeds and juveniles move to the next stage in one time step (for irises the time step can be chosen to be one year [10]). We consider the following three-stage discrete model:

$$
\left\{\begin{array}{l}
x_{t+1}=b_{1} z_{t} \\
y_{t+1}=s_{1} x_{t}+b_{2} z_{t} \\
z_{t+1}=s_{2}\left(y_{t}+z_{t}\right) y_{t}+s_{3}\left(y_{t}+z_{t}\right) z_{t} \\
\left(x_{0}, y_{0}, z_{0}\right) \in \mathbb{R}_{+}^{3} \backslash(0,0,0) .
\end{array}\right.
$$

We assume $s_{i}, i=1,2,3$ have the following properties: 


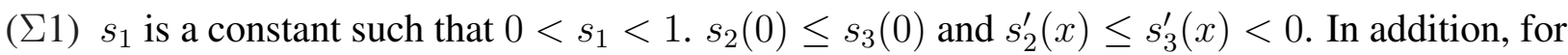
$i=2,3, s_{i}(x) \in C^{1}[0, \infty),\left(s_{i}(x) x\right)^{\prime}>0, \lim _{x \rightarrow \infty} s_{i}(x)=0, \lim _{x \rightarrow \infty} s_{i}(x) x=\hat{a}_{i}<\infty$, and $s_{i}(0)=a_{i}\left(0<a_{i}<1\right)$.

Note that $(\Sigma 1)$ implies that $s_{2}(x) \leq s_{3}(x)$. Also, $(\Sigma 1)$ is satisfied by the following Beverton-Holt dynamics [3] which were utilized in [10]:

$$
s_{i}(x)=\frac{a_{i}}{1+k_{i} x}, i=2,3,
$$

provided that $a_{2} \leq a_{3}$ and $k_{3} \leq k_{2}$.

In this section, we prove that if the inherent net reproductive number is greater than one, then system (2.1) has a unique interior equilibrium, which is globally asymptotically stable. We begin by finding the inherent net reproductive number. We first rewrite the system in the following matrix form:

$$
X_{t+1}=M\left(X_{t}\right) X_{t}
$$

where

$$
M(X)=\left(\begin{array}{ccc}
0 & 0 & b_{1} \\
s_{1} & 0 & b_{2} \\
0 & s_{2}(y+z) & s_{3}(y+z)
\end{array}\right)
$$

Hence,

$$
X \leq Y \text { implies } M(X) \geq M(Y),
$$

where the vector and matrix inequalities hold componentwise.

Then the inherent projection matrix [5] can be written as follows:

$$
M(0)=\left(\begin{array}{ccc}
0 & 0 & b_{1} \\
s_{1} & 0 & b_{2} \\
0 & a_{2} & a_{3}
\end{array}\right)=G+F
$$

where

$$
G=\left(\begin{array}{ccc}
0 & 0 & 0 \\
s_{1} & 0 & 0 \\
0 & a_{2} & a_{3}
\end{array}\right)
$$

and

$$
F=\left(\begin{array}{ccc}
0 & 0 & b_{1} \\
0 & 0 & b_{2} \\
0 & 0 & 0
\end{array}\right)
$$

By $[4,5,7]$, the inherent net reproductive number $R_{0}$ is the positive, simple, and strictly dominant eigenvalue of the next generation matrix $F(I-G)^{-1}$. Thus, we have

$$
R_{0}:=\frac{b_{1} s_{1} a_{2}+a_{2} b_{2}}{1-a_{3}} .
$$


Lemma 1. There exists a compact set $\mathcal{K} \in \mathbb{R}_{+}^{3}$ such that every forward solution sequence of (2.1) enters $\mathcal{K}$ in at most three time steps, and remains in $\mathcal{K}$ ever after.

Proof. From $(\Sigma 1)$, we have that

$$
\begin{aligned}
z_{t+1} & =s_{2}\left(y_{t}+z_{t}\right) y_{t}+s_{3}\left(y_{t}+z_{t}\right) z_{t} \\
& \leq s_{3}\left(y_{t}+z_{t}\right) y_{t}+s_{3}\left(y_{t}+z_{t}\right) z_{t} \\
& =s_{3}\left(y_{t}+z_{t}\right)\left(y_{t}+z_{t}\right) \\
& \leq \hat{a}_{3}, \forall t=0,1, \ldots
\end{aligned}
$$

It follows that

$$
z_{t} \leq \hat{a}_{3}, \forall t=1,2, \ldots
$$

Therefore,

$$
x_{t+1}=b_{1} z_{t} \leq b_{1} \hat{a}_{3}, \forall t=1,2, \ldots
$$

and hence

$$
x_{t} \leq b_{1} \hat{a}_{3}, \forall t=2,3, \ldots
$$

Similarly,

$$
y_{t+1}=s_{1} x_{t}+b_{2} z_{t} \leq s_{1} b_{1} \hat{a}_{3}+b_{2} \hat{a}_{3}, \forall t=2,3, \ldots
$$

and

$$
y_{t} \leq\left(s_{1} b_{1}+b_{2}\right) \hat{a}_{3}, \forall t=3,4, \ldots
$$

Define $\mathcal{K}=\left\{(x, y, z) \in \mathbb{R}_{+}^{3} \mid x \in\left[0, b_{1} \hat{a}_{3}\right], y \in\left[0,\left(s_{1} b_{1}+b_{2}\right) \hat{a}_{3}\right], z \in\left[0, \hat{a}_{3}\right]\right\}$, then every forward solution sequence enters $\mathcal{K}$ in at most three time steps and stays in $\mathcal{K}$ forever after.

We now state the following theorem for the global dynamics of (2.1):

Theorem 1. Concerning the system (2.1) we have the following:

a) If $R_{0}<1$, then system (2.1) only has the trivial equilibrium $E_{0}=(0,0,0)$ which is globally asymptotically stable in $\mathbb{R}_{+}^{3} \backslash(0,0,0)$.

b) If $R_{0}>1$, then $E_{0}$ is unstable and system (2.1) has a unique interior equilibrium $E_{1}=$ $(\hat{x}, \hat{y}, \hat{z})$ which is globally asymptotically stable in $\mathbb{R}_{+}^{3} \backslash(0,0,0)$.

Proof. a) Let $R_{0}<1$. Clearly the model (2.1) has no boundary equilibrium other than the trivial one. Suppose that $(\hat{x}, \hat{y}, \hat{z})$ is an interior equilibrium of (2.1). Then this equilibrium must satisfy

$$
1=b_{1} s_{1} s_{2}(y+z)+b_{2} s_{2}(y+z)+s_{3}(y+z)=: F(y+z) .
$$

Notice that $F(y+z)$ is a strictly decreasing function of $y+z$ by $(\Sigma 1)$, with $F(0)=b_{1} s_{1} a_{2}+$ $b_{2} a_{2}+a_{3}$, and $\lim _{\phi \rightarrow \infty} F(\phi)=0$. Thus, equation (2.3) has a unique positive solution if and only if

$$
b_{1} s_{1} a_{2}+b_{2} a_{2}+a_{3}>1 \text {. }
$$


From this and (2.1) it follows that the interior equilibrium is unique whenever it exists. Note that the above inequality is equivalent to

$$
R_{0}=\frac{b_{1} s_{1} a_{2}+a_{2} b_{2}}{1-a_{3}}>1
$$

Therefore, since $R_{0}<1$, there is no nontrivial equilibrium for system (2.1).

Notice that the inherent projection matrix $M(0)$ of system (2.1) is nonnegative, irreducible, and primitive, it follows from [5] (Theorem 1.1.3, p.10) that it has a positive, simple, and strictly dominant eigenvalue $r$. And since $R_{0}<1$, we have $r<1$. Hence, $\lim _{t \rightarrow \infty} M^{t}(0)=0$. So with any initial value $X_{0}>0$, we have that $0 \leq X_{1}=M\left(X_{0}\right) X_{0} \leq M(0) X_{0}$ by (2.2). Repeating this gives that $0 \leq X_{t} \leq M^{t}(0) X_{0}$. Since $\lim _{t \rightarrow \infty} M^{t}(0) X_{0}=0$, we have $\lim _{t \rightarrow \infty} X_{t}=0$.

b) Suppose $R_{0}>1$. By Theorem 1.1.3 [5], $M(0)$ has a positive, strictly dominant eigenvalue greater than 1. Thus, $E_{0}$ is unstable. Clearly, from the argument in part a) the model (2.1) has a unique interior equilibrium $E_{1}=(\hat{x}, \hat{y}, \hat{z})$. We now show that $E_{1}$ is locally asymptotically stable. Using the short notation $s_{i}$ instead of $s_{i}(\hat{y}+\hat{z})$ and $s_{i}^{\prime}$ instead of $s_{i}^{\prime}(\hat{y}+\hat{z}), i=2,3$, we see that the linearized system of (2.1) at $E_{1}$ has a coefficient matrix

$$
J\left(E_{1}\right)=\left(\begin{array}{ccc}
0 & 0 & b_{1} \\
s_{1} & 0 & b_{2} \\
0 & s_{2}^{\prime} \hat{y}+s_{2}+s_{3}^{\prime} \hat{z} & s_{2}^{\prime} \hat{y}+s_{3}^{\prime} \hat{z}+s_{3}
\end{array}\right) .
$$

Let $J_{13}=b_{1}, J_{21}=s_{1}, J_{23}=b_{2}, J_{32}=s_{2}^{\prime} \hat{y}+s_{2}+s_{3}^{\prime} \hat{z}$ and $J_{33}=s_{2}^{\prime} \hat{y}+s_{3}^{\prime} \hat{z}+s_{3}$. Then the eigenvalues $\lambda$ of $J\left(E_{1}\right)$ satisfy $p_{1}(\lambda)=\lambda^{3}-J_{33} \lambda^{2}-J_{23} J_{32} \lambda-J_{13} J_{21} J_{32}=0$. So applying the Jury conditions $[2,8]$, and using $1=b_{1} s_{1} s_{2}+b_{2} s_{2}+s_{3}$ we have the following:

$$
\begin{aligned}
p_{1}(1)= & 1-J_{33}-J_{23} J_{32}-J_{13} J_{21} J_{32} \\
= & b_{1} s_{1} s_{2}+b_{2} s_{2}+s_{3}-s_{2}^{\prime} \hat{y}-s_{3}^{\prime} \hat{z}-s_{3}-b_{2}\left(s_{2}^{\prime} \hat{y}+s_{2}+s_{3}^{\prime} \hat{z}\right) \\
& -b_{1} s_{1}\left(s_{2}^{\prime} \hat{y}+s_{2}+s_{3}^{\prime} \hat{z}\right) \\
= & -s_{2}^{\prime} \hat{y}-s_{3}^{\prime} \hat{z}-b_{2}\left(s_{2}^{\prime} \hat{y}+s_{3}^{\prime} \hat{z}\right)-b_{1} s_{1}\left(s_{2}^{\prime} \hat{y}+s_{3}^{\prime} \hat{z}\right)
\end{aligned}
$$

and

$$
\begin{aligned}
p_{1}(-1)= & -1-J_{33}+J_{23} J_{32}-J_{13} J_{21} J_{32} \\
= & -b_{1} s_{1} s_{2}-b_{2} s_{2}-s_{3}-s_{2}^{\prime} \hat{y}-s_{3}^{\prime} \hat{z}-s_{3}+b_{2}\left(s_{2}^{\prime} \hat{y}+s_{2}+s_{3}^{\prime} \hat{z}\right) \\
& -b_{1} s_{1}\left(s_{2}^{\prime} \hat{y}+s_{2}+s_{3}^{\prime} \hat{z}\right) \\
= & -b_{1} s_{1}\left(s_{2}+s_{3}^{\prime} \hat{z}\right)-\left(s_{3}+s_{2}^{\prime} \hat{y}\right)-\left(s_{3}^{\prime} \hat{z}+s_{3}\right) \\
& +b_{2}\left(s_{2}^{\prime} \hat{y}+s_{3}^{\prime} \hat{z}\right)-b_{1} s_{1}\left(s_{2}^{\prime} \hat{y}+s_{2}\right) .
\end{aligned}
$$

By $(\Sigma 1), s_{i}^{\prime}<0$, for $i=2,3$. Hence, $p_{1}(1)>0$. Also, by $(\Sigma 1)$, we know that $s_{2} \leq s_{3}$ and $\left(s_{i}(x) x\right)^{\prime}>0$, for $i=2,3$. So $s_{2}^{\prime} \hat{y}+s_{2}^{\prime} \hat{z}+s_{2}>0$ and $s_{3}^{\prime} \hat{y}+s_{3}^{\prime} \hat{z}+s_{3}>0$. It follows that $s_{3}+s_{3}^{\prime} \hat{z} \geq s_{2}+s_{3}^{\prime} \hat{z} \geq s_{2}+s_{2}^{\prime} \hat{z}>0$ and $s_{3}+s_{2}^{\prime} \hat{y} \geq s_{2}+s_{2}^{\prime} \hat{y}>0$. Thus, $p_{1}(-1)<0$.

Now, we proceed to verify the last inequality of Jury conditions: $1-\left(J_{13} J_{21} J_{32}\right)^{2}-\mid J_{23} J_{32}+$ $J_{13} J_{21} J_{32} J_{33} \mid>0$. Replacing 1 by the square of $b_{1} s_{1} s_{2}+b_{2} s_{2}+s_{3}$ gives

$$
1=b_{1}^{2} s_{1}^{2} s_{2}^{2}+2 b_{1} s_{1} s_{2}\left(b_{2} s_{2}+s_{3}\right)+b_{2}^{2} s_{2}^{2}+2 b_{2} s_{2} s_{3}+s_{3}^{2} .
$$


Thus,

$$
\begin{aligned}
& 1-\left(J_{13} J_{21} J_{32}\right)^{2}-\left|J_{23} J_{32}+J_{13} J_{21} J_{32} J_{33}\right| \\
= & \left(b_{1} s_{1} s_{2}+b_{2} s_{2}+s_{3}\right)^{2}-b_{1}^{2} s_{1}^{2}\left(s_{2}^{\prime} \hat{y}+s_{2}+s_{3}^{\prime} \hat{z}\right)^{2}-b_{2}\left(s_{2}^{\prime} \hat{y}+s_{2}+s_{3}^{\prime} \hat{z}\right) \\
& -b_{1} s_{1}\left(s_{2}^{\prime} \hat{y}+s_{2}+s_{3}^{\prime} \hat{z}\right)\left(s_{2}^{\prime} \hat{y}+s_{3}+s_{3}^{\prime} \hat{z}\right) \\
= & b_{1}^{2} s_{1}^{2} s_{2}^{2}+2 b_{1} s_{1} s_{2}\left(b_{2} s_{2}+s_{3}\right)+b_{2}^{2} s_{2}^{2}+2 b_{2} s_{2} s_{3}+s_{3}^{2}-b_{1}^{2} s_{1}^{2}\left(s_{2}^{\prime} \hat{y}+s_{2}+s_{3}^{\prime} \hat{z}\right)^{2} \\
& -b_{1} s_{1}\left[\left(s_{2}^{\prime} \hat{y}+s_{3}^{\prime} \hat{z}\right)^{2}+s_{2} s_{3}+s_{2}\left(s_{2}^{\prime} \hat{y}+s_{3}^{\prime} \hat{z}\right)+s_{3}\left(s_{2}^{\prime} \hat{y}+s_{3}^{\prime} \hat{z}\right)\right] \\
& -b_{2} s_{2}-b_{2}\left(s_{2}^{\prime} \hat{y}+s_{3}^{\prime} \hat{z}\right) \\
= & b_{1}^{2} s_{1}^{2} s_{2}^{2}-b_{1}^{2} s_{1}^{2}\left(s_{2}^{\prime} \hat{y}+s_{2}+s_{3}^{\prime} \hat{z}\right)^{2}+b_{1} s_{1} s_{2} s_{3}-b_{1} s_{1}\left(s_{2}^{\prime} \hat{y}+s_{3}^{\prime} \hat{z}\right)^{2} \\
& +b_{2} s_{2}\left(2 b_{1} s_{1} s_{2}+b_{2} s_{2}+2 s_{3}-1\right)-b_{1} s_{1}\left[s_{2}\left(s_{2}^{\prime} \hat{y}+s_{3}^{\prime} \hat{z}\right)+s_{3}\left(s_{2}^{\prime} \hat{y}+s_{3}^{\prime} \hat{z}\right)\right] \\
& +s_{3}^{2}-b_{2}\left(s_{2}^{\prime} \hat{y}+s_{3}^{\prime} \hat{z}\right) .
\end{aligned}
$$

Since $1>s_{2}>s_{2}^{\prime} \hat{y}+s_{2}+s_{3}^{\prime} \hat{z} \geq s_{2}^{\prime} \hat{y}+s_{2}+s_{2}^{\prime} \hat{z}>0$, it follows that $b_{1}^{2} s_{1}^{2} s_{2}^{2}>b_{1}^{2} s_{1}^{2}\left(s_{2}^{\prime} \hat{y}+s_{2}+s_{3}^{\prime} \hat{z}\right)^{2}$. Also, since $s_{2}>\left|s_{2}^{\prime} \hat{y}+s_{3}^{\prime} \hat{z}\right|$ and $s_{3}>\left|s_{2}^{\prime} \hat{y}+s_{3}^{\prime} \hat{z}\right|$, then $s_{2} s_{3}>\left(s_{2}^{\prime} \hat{y}+s_{3}^{\prime} \hat{z}\right)^{2}$. So $b_{1} s_{1} s_{2} s_{3}-$ $b_{1} s_{1}\left(s_{2}^{\prime} \hat{y}+s_{3}^{\prime} \hat{z}\right)^{2}=b_{1} s_{1}\left[s_{2} s_{3}-\left(s_{2}^{\prime} \hat{y}+s_{3}^{\prime} \hat{z}\right)^{2}\right]>0$. Moreover, using $1=b_{1} s_{1} s_{2}+b_{2} s_{2}+s_{3}$ gives $b_{2} s_{2}\left(2 b_{1} s_{1} s_{2}+b_{2} s_{2}+2 s_{3}-1\right)=b_{2} s_{2}\left(b_{1} s_{1} s_{2}+s_{3}\right)>0$. Note the rest terms are all positive. So the above expression is positive. Therefore, $E_{1}$ is locally asymptotically stable.

Next, we show that $E_{1}$ is globally attracting by using a similar approach as in [1]. Since every solution starting on the boundary of $\mathbb{R}_{+}^{3} \backslash(0,0,0)$ enters the positively invariant set $\operatorname{Int}\left(\mathbb{R}_{+}^{3}\right)$ in at most 3 time steps, it suffices to prove the global asymptotic stability for solutions in $\operatorname{Int}\left(\mathbb{R}_{+}^{3}\right)$. Also by Lemma 1 , every solution sequence enters $\mathcal{K}$ in at most three time steps and stays in $\mathcal{K}$. So we can consider $X_{0}=\left(x_{0}, y_{0}, z_{0}\right) \in \operatorname{Int}\left(\mathbb{R}_{+}^{3}\right) \cap \mathcal{K}$. Clearly, the unique positive equilibrium $E_{1}=(\hat{x}, \hat{y}, \hat{z})$ is in $\mathcal{K}$.

Define $b:=\sup \mathcal{K}$ (the maximal element in $\mathcal{K}$ ). So by Lemma $1, T(b) \leq b$, where $T$ denotes the right hand side of (2.1), i.e., $T(X)=M(X) X$. We say a map $f: \mathbb{R}^{n} \rightarrow \mathbb{R}^{n}$ is monotone if $X \leq Y$ implies $f(X) \leq f(Y)$. Clearly the Jacobian matrix of the map $T$ is given by

$$
D(T(X))=\left(\begin{array}{ccc}
0 & 0 & b_{1} \\
s_{1} & 0 & b_{2} \\
0 & s_{2}^{\prime} y+s_{2}+s_{3}^{\prime} z & s_{2}^{\prime} y+s_{3}^{\prime} z+s_{3}
\end{array}\right) .
$$

Note that $s_{2}^{\prime} y+s_{3}^{\prime} z+s_{3} \geq s_{2}^{\prime} y+s_{2}+s_{3}^{\prime} z \geq s_{2}^{\prime} y+s_{2}+s_{2}^{\prime} z=\left(s_{2}(y+z)(y+z)\right)^{\prime}>0$. Thus, since the elements of $D(T(X))$ are all nonnegative, we have that the map $T$ is monotone.

Since $M(0)$ is irreducible and nonnegative, its spectral radius $r(>1)$ is an eigenvalue with a corresponding positive eigenvector $v$ such that $M(0) v=r v$. Since $r>1$, for all $\epsilon>0$ small enough, we have $T(\epsilon v)=M(\epsilon v) \epsilon v=r \epsilon v+o(\epsilon) \geq \epsilon v$. Then for any given $X_{0} \in \operatorname{Int}\left(\mathbb{R}_{+}^{3}\right) \cap \mathcal{K}$, we can choose a sufficiently small $\epsilon>0$ such that $a:=\epsilon v \leq X_{0}$, i.e. $a \leq T(a)$. So by Lemma 2 in the Appendix, $E_{1}$ is globally asymptotically stable.

\section{Two-Species Model and Competitive Exclusion}

In this section, we are interested in investigating the interaction between two similar species. We assume that each of the species can be modeled by a stage-structured model of the form (2.1). For 
convenience we let $\phi_{t}^{A}=y_{t}^{A}+z_{t}^{A}$ be the total number of plants (juveniles and adults) for species $A$, $\phi_{t}^{B}=y_{t}^{B}+z_{t}^{B}$ be the total number of plants for species $B$, and $\phi_{t}=\phi_{t}^{A}+\phi_{t}^{B}$ be the total number of plants for both species. We assume that due to competition, the survivorship of juveniles and adults for both species depend on $\phi$, the total number of plants. This leads to the following six difference equation two-species model:

$$
\left\{\begin{array}{l}
x_{t+1}^{A}=b_{1}^{A} z_{t}^{A} \\
y_{t+1}^{A}=s_{1}^{A} x_{t}^{A}+b_{2}^{A} z_{t}^{A} \\
z_{t+1}^{A}=s_{2}^{A}\left(\phi_{t}\right) y_{t}^{A}+s_{3}^{A}\left(\phi_{t}\right) z_{t}^{A} \\
x_{t+1}^{B}=b_{1}^{B} z_{t}^{B} \\
y_{t+1}^{B}=s_{1}^{B} x_{t}^{B}+b_{2}^{B} z_{t}^{B} \\
z_{t+1}^{B}=s_{2}^{B}\left(\phi_{t}\right) y_{t}^{B}+s_{3}^{B}\left(\phi_{t}\right) z_{t}^{B} \\
\left(x_{0}^{A}, y_{0}^{A}, z_{0}^{A}\right) \in \mathbb{R}_{+}^{3} \backslash(0,0,0) \\
\left(x_{0}^{B}, y_{0}^{B}, z_{0}^{B}\right) \in \mathbb{R}_{+}^{3} \backslash(0,0,0) .
\end{array}\right.
$$

The first three equations describe the dynamics of the species $A$ and the next three equations, which have the same structure as the first three, model the dynamics of species $B$. The coupling between the two species is due to the dependency of $s_{2}^{A}, s_{3}^{A}, s_{2}^{B}$ and $s_{3}^{B}$ on the total number of plants, $\phi$, of both species $A$ and $B$. Here, $s_{i}^{A}, i=2,3$ satisfy assumption $(\Sigma 1)$ with $a_{i}$ replaced by $a_{i}^{A}$ and $\hat{a}_{i}$ replaced with $\hat{a}_{i}^{A}$. Similarly, $s_{i}^{B}, i=2,3$ satisfy assumption $(\Sigma 1)$ with $a_{i}$ replaced by $a_{i}^{B}$ and $\hat{a}_{i}$ replaced with $\hat{a}_{i}^{B}$.

We define the net reproductive number of species $A$ and $B$ at density level $\phi$ by

$$
R^{A}(\phi)=\frac{b_{1}^{A} s_{1}^{A} s_{2}^{A}(\phi)+s_{2}^{A}(\phi) b_{2}^{A}}{1-s_{3}^{A}(\phi)},
$$

and

$$
R^{B}(\phi)=\frac{b_{1}^{B} s_{1}^{B} s_{2}^{B}(\phi)+s_{2}^{B}(\phi) b_{2}^{B}}{1-s_{3}^{B}(\phi)} .
$$

If we denote the inherent net reproductive number of species $A$ by $R_{0}^{A}$ and of species $B$ by $R_{0}^{B}$, then $R_{0}^{A}=R^{A}(0)$ and $R_{0}^{B}=R^{B}(0)$.

We define

$$
F^{A}(\phi)=b_{1}^{A} s_{1}^{A} s_{2}^{A}(\phi)+s_{2}^{A}(\phi) b_{2}^{A}+s_{3}^{A}(\phi)
$$

and

$$
F^{B}(\phi)=b_{1}^{B} s_{1}^{B} s_{2}^{B}(\phi)+s_{2}^{B}(\phi) b_{2}^{B}+s_{3}^{B}(\phi) .
$$

Notice that $F^{A}(\phi)$ and $F^{B}(\phi)$ are decreasing functions with

$$
\lim _{\phi \rightarrow \infty} F^{A}(\phi)=0 \text { and } \lim _{\phi \rightarrow \infty} F^{B}(\phi)=0 .
$$

Furthermore, $F^{A}(0)>1$ if and only if $R_{0}^{A}>1$, and $F^{B}(0)>1$ if and only if $R_{0}^{B}>1$. Thus, if $R_{0}^{A}>1$ and $R_{0}^{B}>1$ then there exist unique $\hat{\phi}^{A}$ and $\hat{\phi}^{B}$ such that $F^{A}\left(\hat{\phi}^{A}\right)=1$ and $F^{B}\left(\hat{\phi}^{B}\right)=1$. 
Clearly, model (3.1) has the trivial equilibrium denoted by $E_{0}=(0,0,0,0,0,0)$. Furthermore, if $R_{0}^{A}>1$ and $R_{0}^{B}>1$, then from Section 2, we know each species (living alone) has a positive (globally asymptotically stable) fixed point. Denote this equilibrium for species $A$ by $E^{A}=\left(\hat{x}^{A}, \hat{y}^{A}, \hat{z}^{A}\right)$ and for species $B$ by $E^{B}=\left(\hat{x}^{B}, \hat{y}^{B}, \hat{z}^{B}\right)$. Thus, it follows that model (3.1) has two nontrivial boundary equilibria given by $E_{1}=\left(E^{A}, 0\right) \in \mathbb{R}_{+}^{6}$ and $E_{2}=\left(0, E^{B}\right) \in \mathbb{R}_{+}^{6}$. Note that in this case $\hat{\phi}^{A}=\hat{y}^{A}+\hat{z}^{A}$ for $E_{1}$ and $\hat{\phi}^{B}=\hat{y}^{B}+\hat{z}^{B}$ for $E_{2}$. We now have the following theorem:

Theorem 2. Assume that $R_{0}^{A}>1$ and $R_{0}^{B}>1$. Concerning the system (3.1) we have the following:

a) If $\hat{\phi}^{A}>\hat{\phi}^{B}$, then $E_{1}=\left(E^{A}, 0\right)$ is locally asymptotically stable and $E_{2}=\left(0, E^{B}\right)$ is unstable.

b) If $\hat{\phi}^{A}<\hat{\phi}^{B}$, then $E_{2}=\left(0, E^{B}\right)$ is locally asymptotically stable and $E_{1}=\left(E^{A}, 0\right)$ is unstable.

c) If $\hat{\phi}^{A}=\hat{\phi}^{B}$, then there exists an interior equilibrium.

Proof. a) The linearized system of (3.1) at $E_{1}$ has the coefficient matrix

$$
J\left(E_{1}\right)=\left(\begin{array}{cc}
A_{1} & C_{1} \\
0 & B_{1}
\end{array}\right)
$$

where

$$
\begin{gathered}
A_{1}=\left(\begin{array}{ccc}
0 & 0 & b_{1}^{A} \\
s_{1}^{A} & 0 & b_{2}^{A} \\
0 & s_{2}^{\prime A}\left(\hat{\phi}^{A}\right) \hat{y}^{A}+s_{2}^{A}\left(\hat{\phi}^{A}\right)+s_{3}^{\prime A}\left(\hat{\phi}^{A}\right) \hat{z}^{A} & s_{2}^{\prime A}\left(\hat{\phi}^{A}\right) \hat{y}^{A}+s_{3}^{\prime A}\left(\hat{\phi}^{A}\right) \hat{z}^{A}+s_{3}^{A}\left(\hat{\phi}^{A}\right)
\end{array}\right) \\
B_{1}=\left(\begin{array}{ccc}
0 & 0 & b_{1}^{B} \\
s_{1}^{B} & 0 & b_{2}^{B} \\
0 & s_{2}^{B}\left(\hat{\phi}^{A}\right) & s_{3}^{B}\left(\hat{\phi}^{A}\right)
\end{array}\right)
\end{gathered}
$$

and

$$
C_{1}=\left(\begin{array}{ccc}
0 & 0 & 0 \\
0 & 0 & 0 \\
0 & s_{2}^{\prime A}\left(\hat{\phi}^{A}\right) \hat{y}^{A}+s_{3}^{\prime A}\left(\hat{\phi}^{A}\right) \hat{z}^{A} & s_{2}^{\prime A}\left(\hat{\phi}^{A}\right) \hat{y}^{A}+s_{3}^{\prime A}\left(\hat{\phi}^{A}\right) \hat{z}^{A}
\end{array}\right)
$$

Similarly, the linearized system at $E_{2}$ has the coefficient matrix

$$
J\left(E_{2}\right)=\left(\begin{array}{cc}
A_{2} & 0 \\
C_{2} & B_{2}
\end{array}\right)
$$

where

$$
A_{2}=\left(\begin{array}{ccc}
0 & 0 & b_{1}^{A} \\
s_{1}^{A} & 0 & b_{2}^{A} \\
0 & s_{2}^{A}\left(\hat{\phi}^{B}\right) & s_{3}^{A}\left(\hat{\phi}^{B}\right)
\end{array}\right)
$$




$$
B_{2}=\left(\begin{array}{ccc}
0 & 0 & b_{1}^{B} \\
s_{1}^{B} & 0 & b_{2}^{B} \\
0 & s_{2}^{\prime B}\left(\hat{\phi}^{B}\right) \hat{y}^{B}+s_{2}^{B}\left(\hat{\phi}^{B}\right)+s_{3}^{\prime B}\left(\hat{\phi}^{B}\right) \hat{z}^{B} & s_{2}^{B}\left(\hat{\phi}^{B}\right) \hat{y}^{B}+s_{3}^{\prime B}\left(\hat{\phi}^{B}\right) \hat{z}^{B}+s_{3}^{B}\left(\hat{\phi}^{B}\right)
\end{array}\right)
$$

and

$$
C_{2}=\left(\begin{array}{ccc}
0 & 0 & 0 \\
0 & 0 & 0 \\
0 & s_{2}^{\prime B}\left(\hat{\phi}^{B}\right) \hat{y}^{B}+s_{3}^{\prime B}\left(\hat{\phi}^{B}\right) \hat{z}^{B} & s_{2}^{B}\left(\hat{\phi}^{B}\right) \hat{y}^{B}+s_{3}^{B}\left(\hat{\phi}^{B}\right) \hat{z}^{B}
\end{array}\right) .
$$

Since $R_{0}^{A}>1$, it follows by arguments as those in Section 2 that the eigenvalues of $A_{1}$ are less than 1 in magnitude. Also, since $\hat{\phi}^{A}>\hat{\phi}^{B}$, then $F^{B}\left(\hat{\phi}^{A}\right)<1$, which implies

$$
R^{B}\left(\hat{\phi}^{A}\right)=\frac{b_{1}^{B} s_{1}^{B} s_{2}^{B}\left(\hat{\phi}^{A}\right)+s_{2}^{B}\left(\hat{\phi}^{A}\right) b_{2}^{B}}{1-s_{3}^{B}\left(\hat{\phi}^{A}\right)}<1
$$

So the positive strictly dominant eigenvalue of $B_{1}$ is less than 1 . Hence, $E_{1}=\left(E^{A}, 0\right)$ is locally asymptotically stable.

We now show that $E_{2}=\left(0, E^{B}\right)$ is unstable. Since $\hat{\phi}^{A}>\hat{\phi}^{B}$, we have $F^{A}\left(\hat{\phi}^{B}\right)>F^{A}\left(\hat{\phi}^{A}\right)=1$. Thus,

$$
R^{A}\left(\hat{\phi}^{B}\right)=\frac{b_{1}^{A} s_{1}^{A} s_{2}^{A}\left(\hat{\phi}^{B}\right)+s_{2}^{A}\left(\hat{\phi}^{B}\right) b_{2}^{A}}{1-s_{3}^{A}\left(\hat{\phi}^{B}\right)}>1,
$$

which implies that there exists an eigenvalue of $A_{2}$ with magnitude larger than one. Thus, $E_{2}$ is unstable.

b) Follows by similar arguments as in $a$ ).

c) From the model (3.1) to have an interior equilibrium the following must hold: there exists a $\phi$ such that

$$
F^{A}(\phi)=1, \text { and } F^{B}(\phi)=1,
$$

which clearly holds since $\hat{\phi}^{A}=\hat{\phi}^{B}$. We can also see that the $\phi$ is not unique and there is a continuum of interior equilibria.

By Theorem 2, we see that if $\hat{\phi}^{A} \neq \hat{\phi}^{B}$ then either $E_{1}$ or $E_{2}$ is locally asymptotically stable. We now show that such a stability is indeed global.

Theorem 3. Suppose $R_{0}^{A}>1$ and $R_{0}^{B}>1$. Concerning the system (3.1) we have the following:

a) If $\hat{\phi}^{A}>\hat{\phi}^{B}$, then $E_{1}=\left(E^{A}, 0\right)$ is a globally asymptotically stable equilibrium in $\left\{\left(X^{A}, X^{B}\right)\right.$ $\in \mathbb{R}_{+}^{3} \times \mathbb{R}_{+}^{3}: X^{A} \neq 0$ and $\left.X^{B} \neq 0\right\}$.

b) If $\hat{\phi}^{A}<\hat{\phi}^{B}$, then $E_{2}=\left(0, E^{B}\right)$ is a globally asymptotically stable equilibrium in $\left\{\left(X^{A}, X^{B}\right)\right.$ $\in \mathbb{R}_{+}^{3} \times \mathbb{R}_{+}^{3}: X^{A} \neq 0$ and $\left.X^{B} \neq 0\right\}$. 
Proof. a) System (3.1) can be written in the matrix form:

$$
X_{t+1}=M\left(X_{t}\right) X_{t}=T\left(X_{t}\right) .
$$

Here, $X_{t}=\left(X_{t}^{A}, X_{t}^{B}\right)^{t r}, X_{t}^{A}=\left(x_{t}^{A}, y_{t}^{A}, z_{t}^{A}\right)^{t r}, X_{t}^{B}=\left(x_{t}^{B}, y_{t}^{B}, z_{t}^{B}\right)^{t r}$, and $t r$ denotes the transpose of a vector. Also, the projection matrix is

$$
M(X)=\left(\begin{array}{cc}
M^{A}(X) & 0 \\
0 & M^{B}(X)
\end{array}\right),
$$

where

$$
M^{A}(X)=\left(\begin{array}{ccc}
0 & 0 & b_{1}^{A} \\
s_{1}^{A} & 0 & b_{2}^{A} \\
0 & s_{2}^{A}(\phi) & s_{3}^{A}(\phi)
\end{array}\right)
$$

and

$$
M^{B}(X)=\left(\begin{array}{ccc}
0 & 0 & b_{1}^{B} \\
s_{1}^{B} & 0 & b_{2}^{B} \\
0 & s_{2}^{B}(\phi) & s_{3}^{B}(\phi)
\end{array}\right) .
$$

Note the map $T: \mathbb{R}_{+}^{6} \rightarrow \mathbb{R}_{+}^{6}$ is continuous. To prove global stability we show that the map $T$ above satisfies the hypotheses (H1)-(H3) and (H4) stated in the Appendix and apply Theorem 4 in the Appendix.

To verify (H1), suppose $X=\left(X^{A}, X^{B}\right)^{t r}=\left(x^{A}, y^{A}, z^{A}, x^{B}, y^{B}, z^{B}\right)^{t r}, \bar{X}=\left(\bar{X}^{A}, \bar{X}^{B}\right)^{t r}=$ $\left(\bar{x}^{A}, \bar{y}^{A}, \bar{z}^{A}, \bar{x}^{B}, \bar{y}^{B}, \bar{z}^{B}\right)^{t r} \in \mathbb{R}_{+}^{6}$ and $X<_{K} \bar{X}$. Then we have

$$
x^{A} \leq \bar{x}^{A}, y^{A} \leq \bar{y}^{A}, z^{A} \leq \bar{z}^{A},
$$

where at least one of the inequalities in (3.8) is strict, and

$$
x^{B} \geq \bar{x}^{B}, y^{B} \geq \bar{y}^{B}, z^{B} \geq \bar{z}^{B},
$$

where at least one of the inequalities in (3.9) is strict.

Let $T(X)=\left(T_{1}(X), \ldots, T_{6}(X)\right)^{t r}$, and $\Delta T_{i}=T_{i}(\bar{X})-T_{i}(X), i=1,2, \ldots, 6$. So we have the following:

$$
\begin{aligned}
& \Delta T_{1}=b_{1}^{A}\left(\bar{z}^{A}-z^{A}\right), \\
& \Delta T_{2}=s_{1}^{A}\left(\bar{x}^{A}-x^{A}\right)+b_{2}^{A}\left(\bar{z}^{A}-z^{A}\right), \\
& \Delta T_{3}=\left[s_{2}^{A}(\bar{\phi}) \bar{y}^{A}+s_{3}^{A}(\bar{\phi}) \bar{z}^{A}\right]-\left[s_{2}^{A}(\phi) y^{A}+s_{3}^{A}(\phi) z^{A}\right], \\
& \Delta T_{4}=b_{1}^{B}\left(\bar{z}^{B}-z^{B}\right), \\
& \Delta T_{5}=s_{1}^{B}\left(\bar{x}^{B}-x^{B}\right)+b_{2}^{B}\left(\bar{z}^{B}-z^{B}\right), \\
& \Delta T_{6}=\left[s_{2}^{B}(\bar{\phi}) \bar{y}^{B}+s_{3}^{B}(\bar{\phi}) \bar{z}^{B}\right]-\left[s_{2}^{B}(\phi) y^{B}+s_{3}^{B}(\phi) z^{B}\right] .
\end{aligned}
$$

In order to prove $T(X)<_{K} T(\bar{X})$, we need to show that $\Delta T_{i} \geq 0$ for $i=1,2,3$ with at least one inequality is strict and $\Delta T_{i} \leq 0$ for $i=4,5,6$ with at least one inequality is strict. By (3.8) and (3.9), it is easy to see that $\Delta T_{1}, \Delta T_{2} \geq 0$ and $\Delta T_{4}, \Delta T_{5} \leq 0$. In fact, if the first or the third inequality in (3.8) is strict, then either $\Delta T_{1}$ or $\Delta T_{2}$ is strictly positive. Similar for $\Delta T_{4}$ and $\Delta T_{5}$. 
Therefore, in what follows, we assume that the second inequality is strict, i.e., $y^{A}<\bar{y}^{A}$. Now we verify that $\Delta T_{3}>0$ (If $y^{B}>\bar{y}^{B}$, then $\Delta T_{6}<0$ can be proved in a similar fashion).

As defined previously, $\phi=\phi^{A}+\phi^{B}$ and $\bar{\phi}=\bar{\phi}^{A}+\bar{\phi}^{B}$, where $\phi^{A}=y^{A}+z^{A}, \phi^{B}=y^{B}+z^{B}$, and $\bar{\phi}^{A}=\bar{y}^{A}+\bar{z}^{A}, \bar{\phi}^{B}=\bar{y}^{B}+\bar{z}^{B}$. Then we have two cases:

Case 1: $\phi<\bar{\phi}$. Then

$$
\begin{aligned}
\Delta T_{3}= & {\left[s_{2}^{A}(\bar{\phi}) \bar{y}^{A}+s_{3}^{A}(\bar{\phi}) \bar{z}^{A}\right]-\left[s_{2}^{A}(\phi) y^{A}+s_{3}^{A}(\phi) z^{A}\right] } \\
= & s_{2}^{A}(\bar{\phi}) \bar{\phi}^{A}-s_{2}^{A}(\bar{\phi}) \bar{z}^{A}+s_{3}^{A}(\bar{\phi}) \bar{z}^{A}-s_{2}^{A}(\phi) \phi^{A}+s_{2}^{A}(\phi) z^{A}-s_{3}^{A}(\phi) z^{A} \\
= & s_{2}^{A}(\bar{\phi}) \bar{\phi}-s_{2}^{A}(\bar{\phi}) \bar{\phi}^{B}-s_{2}^{A}(\bar{\phi}) \bar{z}^{A}+s_{3}^{A}(\bar{\phi}) \bar{z}^{A}-s_{2}^{A}(\phi) \phi+s_{2}^{A}(\phi) \phi^{B} \\
& +s_{2}^{A}(\phi) z^{A}-s_{3}^{A}(\phi) z^{A} \\
= & {\left[s_{2}^{A}(\bar{\phi}) \bar{\phi}-s_{2}^{A}(\phi) \phi\right]+\left[s_{2}^{A}(\phi) \phi^{B}-s_{2}^{A}(\bar{\phi}) \bar{\phi}^{B}\right] } \\
& +\bar{z}^{A}\left[s_{3}^{A}(\bar{\phi})-s_{2}^{A}(\bar{\phi})-\left(s_{3}^{A}(\phi)-s_{2}^{A}(\phi)\right)\right] \\
& +\left(s_{3}^{A}(\phi)-s_{2}^{A}(\phi)\right)\left(\bar{z}^{A}-z^{A}\right) .
\end{aligned}
$$

By $(\Sigma 1),\left(s_{i}(x) x\right)^{\prime}>0, s_{i}^{\prime}(x)<0$. Therefore, $s_{2}^{A}(\bar{\phi}) \bar{\phi}-s_{2}^{A}(\phi) \phi>0$. Also,

$$
s_{2}^{A}(\phi) \phi^{B}-s_{2}^{A}(\bar{\phi}) \bar{\phi}^{B}=\phi^{B}\left(s_{2}^{A}(\phi)-s_{2}^{A}(\bar{\phi})\right)+\left(\phi^{B}-\bar{\phi}^{B}\right) s_{2}^{A}(\bar{\phi})>0 .
$$

And $s_{3}^{A}(\bar{\phi})-s_{2}^{A}(\bar{\phi})-\left(s_{3}^{A}(\phi)-s_{2}^{A}(\phi)\right)=\int_{\phi}^{\bar{\phi}}\left(s_{3}^{A}(\xi)-s_{2}^{A}(\xi)\right)^{\prime} d \xi \geq 0$ since $s_{3}^{\prime A}(\phi) \geq s_{2}^{\prime A}(\phi)$.

The last term $\left(s_{3}^{A}(\phi)-s_{2}^{A}(\phi)\right)\left(\bar{z}^{A}-z^{A}\right) \geq 0$ because $s_{3}^{A}(\phi) \geq s_{2}^{A}(\phi)$ and $\bar{z}^{A} \geq z^{A}$.

Thus, $\Delta T_{3}>0$.

Case 2: $\phi \geq \bar{\phi}$. Then

$$
\begin{aligned}
\Delta T_{3} & =s_{2}^{A}(\bar{\phi}) \bar{y}^{A}+s_{3}^{A}(\bar{\phi}) \bar{z}^{A}-s_{2}^{A}(\phi) y^{A}-s_{3}^{A}(\phi) z^{A} \\
& \geq s_{2}^{A}(\phi) \bar{y}^{A}+s_{3}^{A}(\phi) \bar{z}^{A}-s_{2}^{A}(\phi) y^{A}-s_{3}^{A}(\phi) z^{A} \\
& =s_{2}^{A}(\phi)\left(\bar{y}^{A}-y^{A}\right)+s_{3}^{A}(\phi)\left(\bar{z}^{A}-z^{A}\right) .
\end{aligned}
$$

Using the conditions $\bar{y}^{A}>y^{A}$ and $\bar{z}^{A} \geq z^{A}$, we get $\Delta T_{3}>0$ in this case. Hence, (H1) is satisfied.

For $(\mathrm{H} 2), T(0)=0$ is clear. We now show that $0 \in \mathbb{R}_{+}^{6}$ is a repeller. By (3.5), (3.6) and (3.7),

$$
M(0)=\left(\begin{array}{cc}
M^{A}(0) & 0 \\
0 & M^{B}(0)
\end{array}\right),
$$

where

$$
M^{A}(0)=\left(\begin{array}{ccc}
0 & 0 & b_{1}^{A} \\
s_{1}^{A} & 0 & b_{2}^{A} \\
0 & a_{2}^{A} & a_{3}^{A}
\end{array}\right)
$$

and

$$
M^{B}(0)=\left(\begin{array}{ccc}
0 & 0 & b_{1}^{B} \\
s_{1}^{B} & 0 & b_{2}^{B} \\
0 & a_{2}^{B} & a_{3}^{B}
\end{array}\right)
$$


By assumption, both $R_{0}^{A}>1$ and $R_{0}^{B}>1$. And since $M^{A}(0)$ and $M^{B}(0)$ are non-negative, irreducible and primitive, their dominant eigenvalues $\lambda_{1}, \lambda_{2}(>1)$ have corresponding left eigenvectors $v_{1}, v_{2}>0$, i.e., $v_{1}^{t r} M^{A}(0)=\lambda_{1} v_{1}^{t r}, v_{2}^{t r} M^{B}(0)=\lambda_{2} v_{2}^{t r}$. Notice that

$$
\left(v_{1}^{t r}, v_{2}^{t r}\right) M(0)=\left(v_{1}^{t r}, v_{2}^{t r}\right)\left(\begin{array}{cc}
M^{A}(0) & 0 \\
0 & M^{B}(0)
\end{array}\right)=\left(v_{1}^{t r} M^{A}(0), v_{2}^{t r} M^{B}(0)\right)
$$

Pick $\lambda_{1}^{*} \in\left(1, \lambda_{1}\right), \lambda_{2}^{*} \in\left(1, \lambda_{2}\right)$, such that $v_{1}^{t r} M^{A}(0)-\lambda_{1}^{*} v_{1}^{t r}>0$ and $v_{2}^{t r} M^{B}(0)-\lambda_{2}^{*} v_{2}^{t r}>0$. By continuity of $M(X)$, there exists a neighborhood $U$ of $(0,0) \in \mathbb{R}_{+}^{3} \times \mathbb{R}_{+}^{3}$ such that both $v_{1}^{t r} M^{A}(X)-\lambda_{1}^{*} v_{1}^{t r}>0$ and $v_{2}^{t r} M^{B}(X)-\lambda_{2}^{*} v_{2}^{t r}>0$. Define $P: \mathbb{R}_{+}^{3} \times \mathbb{R}_{+}^{3} \rightarrow \mathbb{R}_{+}$as $P(X)=$ $\left(v_{1}^{t r}, v_{2}^{t r}\right) X$. Then $P(X)=0$ for $X \in U$ if and only if $X=0$, and positive elsewhere in $U$. Furthermore,

$$
\begin{aligned}
P(T(X)) & =\left(v_{1}^{t r}, v_{2}^{t r}\right) T(X) \\
& =\left(v_{1}^{t r}, v_{2}^{t r}\right) M(X) X \\
& =\left(v_{1}^{t r}, v_{2}^{t r}\right)\left(\begin{array}{cc}
M^{A}(X) & 0 \\
0 & M^{B}(X)
\end{array}\right) X \\
& =\left(v_{1}^{t r} M^{A}(X), v_{2}^{t r} M^{B}(X)\right) X \\
& >\left(\lambda_{1}^{*} v_{1}^{t r}, \lambda_{2}^{*} v_{2}^{t r}\right) X \\
& >\left(v_{1}^{t r}, v_{2}^{t r}\right) X \\
& =P(X), \text { for any } X \in U \backslash\{0\} .
\end{aligned}
$$

So the origin $0 \in \mathbb{R}_{+}^{6}$ is a repelling fixed point.

To verify (H3), first note that from Lemma 1 it follows that $T\left(\mathbb{R}_{+}^{3} \times\{0\}\right) \subset \mathbb{R}_{+}^{3} \times\{0\}$ and that $T\left(\{0\} \times \mathbb{R}_{+}^{3}\right) \subset\{0\} \times \mathbb{R}_{+}^{3}$. Moreover, since $R_{0}^{A}, R_{0}^{B}>1$, there exist two nontrivial boundary equilibria of (3.1), denoted by $\left(E^{A}, 0\right)=\left(\hat{x}^{A}, \hat{y}^{A}, \hat{z}^{A}, 0,0,0\right)$ and $\left(0, E^{B}\right)=\left(0,0,0, \hat{x}^{B}, \hat{y}^{B}, \hat{z}^{B}\right) \in$ $\mathbb{R}_{+}^{6}$. That is, there exist $E^{A}=\left(\hat{x}^{A}, \hat{y}^{A}, \hat{z}^{A}\right)$ and $E^{B}=\left(\hat{x}^{B}, \hat{y}^{B}, \hat{z}^{B}\right) \in \mathbb{R}_{+}^{3}$ such that $T\left(\left(E^{A}, 0\right)\right)=$ $\left(E^{A}, 0\right)$ and $T\left(\left(0, E^{B}\right)\right)=\left(0, E^{B}\right)$.

By our discussion in Section 2 , we see that both $E^{A}=\left(\hat{x}^{A}, \hat{y}^{A}, \hat{z}^{A}\right)$ and $E^{B}=\left(\hat{x}^{B}, \hat{y}^{B}, \hat{z}^{B}\right)$ are globally asymptotically stable equilibria of the corresponding one-species model. Thus, (H3) holds.

Finally, we show that (H4)' is satisfied. Notice that for $n \geq 1$,

$$
\begin{aligned}
& T_{1}^{n+1}=b_{1}^{A} T_{3}^{n}, \\
& T_{2}^{n+1}=s_{1}^{A} T_{1}^{n}+b_{2}^{A} T_{3}^{n}, \\
& T_{3}^{n+1}=s_{2}^{A}\left(\phi\left(T^{n}\right)\right) T_{2}^{n}+s_{3}^{A}\left(\phi\left(T^{n}\right)\right) T_{3}^{n}, \\
& T_{4}^{n+1}=b_{1}^{B} T_{6}^{n}, \\
& T_{5}^{n+1}=s_{1}^{B} T_{4}^{n}+b_{2}^{B} T_{6}^{n}, \\
& T_{6}^{n+1}=s_{2}^{B}\left(\phi\left(T^{n}\right)\right) T_{5}^{n}+s_{3}^{B}\left(\phi\left(T^{n}\right)\right) T_{6}^{n},
\end{aligned}
$$

where $T^{n}$ is the $n$-fold composition of $T$ and $\phi\left(T^{n}\right)=T_{2}^{n}+T_{3}^{n}+T_{5}^{n}+T_{6}^{n}$. Suppose that $X<_{K} \bar{X}$ with

$$
x^{A}<\bar{x}^{A}, y^{A}=\bar{y}^{A}, z^{A}=\bar{z}^{A} .
$$


and

$$
x^{B}>\bar{x}^{B}, y^{B}=\bar{y}^{B}, z^{B}=\bar{z}^{B} .
$$

Define

$$
\Delta T_{i}^{n}=\bar{T}_{i}^{n}-T_{i}^{n}
$$

for $i=1,2, \ldots, 6$ and $n \geq 1$. So if $n=1, \Delta T_{1}=\Delta T_{3}=\Delta T_{4}=\Delta T_{6}=0, \Delta T_{2}>0$ and $\Delta T_{5}<0$. Now we see that

$$
\begin{aligned}
& \Delta T_{1}^{2}=b_{1}^{A}\left(\bar{T}_{3}-T_{3}\right)=0 \\
& \Delta T_{2}^{2}=s_{1}^{A}\left(\bar{T}_{1}-T_{1}\right)+b_{2}^{A}\left(\bar{T}_{3}-T_{3}\right)=0 \\
& \Delta T_{3}^{2}=s_{2}^{A}(\phi(\bar{T})) \bar{T}_{2}+s_{3}^{A}(\phi(\bar{T})) \bar{T}_{3}-s_{2}^{A}(\phi(T)) T_{2}-s_{3}^{A}(\phi(T)) T_{3}>0 .
\end{aligned}
$$

Similarly, we have that

$$
\Delta T_{4}^{2}=\Delta T_{5}^{2}=0, \text { and } \Delta T_{6}^{2}<0
$$

Then,

$$
\begin{aligned}
& \Delta T_{1}^{3}=b_{1}^{A}\left(\bar{T}_{3}^{2}-T_{3}^{2}\right)>0, \\
& \Delta T_{2}^{3}=s_{1}^{A}\left(\bar{T}_{1}^{2}-T_{1}^{2}\right)+b_{2}^{A}\left(\bar{T}_{3}^{2}-T_{3}^{2}\right)>0, \\
& \Delta T_{3}^{3}=s_{2}^{A}\left(\phi\left(\bar{T}^{2}\right)\right) \bar{T}_{2}^{2}+s_{3}^{A}\left(\phi\left(\bar{T}^{2}\right)\right) \bar{T}_{3}^{2}-s_{2}^{A}\left(\phi\left(T^{2}\right)\right) T_{2}^{2}-s_{3}^{A}\left(\phi\left(T^{2}\right)\right) T_{3}^{2}>0 .
\end{aligned}
$$

Similarly, we can show that the last three corresponding expressions are negative. Therefore, $T^{3}(\bar{X}) \gg_{K} T^{3}(X)$.

If all the inequalities in (3.8) and (3.9) are strict, then by a similar proof as of (H1), $T(\bar{X}) \gg_{K}$ $T(X)$. Furthermore, it can be shown that $T(\bar{X}) \gg_{K} T(X)$ as long as $\bar{z}^{A}>z^{A}$ and $\bar{z}^{B}<z^{B}$.

Now we assume that $\bar{x}^{A}=x^{A}, \bar{z}^{A}=z^{A}$ and $\bar{y}^{A}>y^{A}$. Then $\Delta T_{3}>0$, and hence

$$
\begin{aligned}
& \Delta T_{1}^{2}=b_{1}^{A}\left(\bar{T}_{3}-T_{3}\right)>0 \\
& \Delta T_{2}^{2}=s_{1}^{A}\left(\bar{T}_{1}-T_{1}\right)+b_{2}^{A}\left(\bar{T}_{3}-T_{3}\right)>0 \\
& \Delta T_{3}^{2}=s_{2}^{A}(\phi(\bar{T})) \bar{T}_{2}+s_{3}^{A}(\phi(\bar{T})) \bar{T}_{3}-s_{2}^{A}(\phi(T)) T_{2}-s_{3}^{A}(\phi(T)) T_{3}>0 .
\end{aligned}
$$

Similarly, we have that $\Delta T_{4}^{2}, \Delta T_{5}^{2}$ and $\Delta T_{6}^{2}<0$. Thus, $T^{2}(\bar{X}) \gg_{K} T^{2}(X)$. From the above argument, it easily follows that if $X, Y \in \mathbb{R}_{+}^{6}$ satisfy $X<_{K} Y$ and either $X$ or $Y$ belongs to $\operatorname{Int}\left(\mathbb{R}_{+}^{6}\right)$, then $T^{3}(X) \ll_{K} T^{3}(Y)$.

Let $X=\left(X_{1}, X_{2}\right) \in \mathbb{R}_{+}^{3} \times \mathbb{R}_{+}^{3}$ satisfy $X_{i} \neq 0, i=1,2$. Then by Lemma 1 , every forward solution sequence of (3.1) enters the positive invariant set $\mathbb{R}_{+}^{3} \times \mathbb{R}_{+}^{3}$ in at most three time steps. So $T^{3}(X) \gg 0$. And this finishes the proof of $(\mathrm{H} 4)^{\prime}$.

Thus, from Theorem 4 it follows that the solutions of (3.1) converge to the boundary equilibrium $E_{1}$ or $E_{2}$.

Now we assume $\hat{\phi}^{A}>\hat{\phi}^{B}$. In order to prove $a$ ), we next show that any solution sequence of system (3.1) will not converge to $E_{2}$. It suffices to prove that $E_{2}$ repels all solutions from the interior. To this end, we want to find a continuous function $H: \mathbb{R}_{+}^{3} \times \mathbb{R}_{+}^{3} \rightarrow \mathbb{R}_{+}$and a neighborhood $V$ of $E_{2}$, such that $H(T(X))>H(X)>0$, for all $X \in V \cap \operatorname{Int}\left(\mathbb{R}_{+}^{6}\right)$ and $H\left(E_{2}\right)=0$.

By (3.2) and (3.6), we see that $M^{A}\left(E_{2}\right)=A_{2}$ is nonnegative, primitive and irreducible. So $M^{A}\left(E_{2}\right)$ has a positive dominant eigenvalue $\hat{\lambda}_{1}$ associated with a left eigenvector $\hat{v}_{1}$ such that 
$\hat{v}_{1}^{t r} M^{A}\left(E_{2}\right)=\hat{\lambda}_{1} \hat{v}_{1}^{\operatorname{tr}}[5]$. Also, from (3.3) we know that $\hat{\lambda}_{1}>1$. Define $H: \mathbb{R}_{+}^{3} \times \mathbb{R}_{+}^{3} \rightarrow$ $\mathbb{R}_{+}$as $H(X)=H\left(\left(X^{A}, X^{B}\right)^{t r}\right)=\hat{v}_{1}^{t r} X^{A}$. Since $\hat{\lambda}_{1}>1$, we can find $\tilde{\lambda}_{1} \in\left(1, \hat{\lambda}_{1}\right)$ such that $\hat{v}_{1}^{t r} M^{A}\left(E_{2}\right)-\tilde{\lambda}_{1} \hat{v}_{1}^{t r}>0$. By continuity of $M^{A}(X)$, there exists a neighborhood $V$ of $E_{2}=$ $\left(0, E^{B}\right) \in \mathbb{R}_{+}^{6}$ such that $\hat{v}_{1}^{t r} M^{A}(X)-\tilde{\lambda}_{1} \hat{v}_{1}^{t r}>0$. Then $H(X)=0$ if and only if $X^{A}=0$ and $H$ is positive elsewhere in $V \cap \operatorname{Int}\left(\mathbb{R}_{+}^{6}\right)$. Also notice that

$$
T(X)=M(X) X=\left(M^{A}(X) X^{A}, M^{B}(X) X^{B}\right)^{\operatorname{tr}} .
$$

Then

$$
\begin{aligned}
H(T(X)) & =H\left(\left(M^{A}(X) X^{A}, M^{B}(X) X^{B}\right)^{t r}\right) \\
& =\hat{v}_{1}^{t r} M^{A}(X) X^{A}>\tilde{\lambda}_{1} \hat{v}_{1}^{t r} X^{A}>\hat{v}_{1}^{t r} X^{A} \\
& =H(X), \text { for any } X \in V \cap\left\{X \in \mathbb{R}_{+}^{6}: X^{A}>0\right\} .
\end{aligned}
$$

Thus, $E_{2}=\left(0, E^{B}\right)$ repels all the solutions from the interior of $\mathbb{R}_{+}^{6}$. Furthermore, since none of the solution sequences could enter the boundary in finite time, this concludes that all the solution sequences with a initial value $\left(X_{0}^{A}, X_{0}^{B}\right)>(0,0)$ will converge to $E_{1}$ provided that $\hat{\phi}^{A}>\hat{\phi}^{B}$, i.e. a) holds.

b) Follows by similar arguments as in $a$ ).

\section{Numerical Results}

In this section, we provide numerical results that corroborate the theoretical ones. We choose parameter values similar to those used in [10] for the blue and yellows iris under fresh and brackish environments. Species $A$ denotes the blue iris population and species $B$ denotes the yellow iris population. We set the time unit to one year [10] and we use the simplified assumption that all seeds germinate in one year and all seedlings become flowering plants in one year. For the graphs in Figure 1 and Figure 2, we let $s_{2}(\phi)=a_{2} /\left(1+k_{2} \phi\right)$ and $s_{3}(\phi)=a_{3} /\left(1+k_{3} \phi\right)$, where $k_{2}=k_{3}=$ 0.003 [10]. The initial conditions are set as $\left(x_{0}^{A}, y_{0}^{A}, z_{0}^{A}\right)=(0,0,1)$ and $\left(x_{0}^{B}, y_{0}^{B}, z_{0}^{B}\right)=(0,0,1)$. [10].

We compute $\hat{\phi}^{A}$ and $\hat{\phi}^{B}$ for the two different environments. In freshwater environment, $\hat{\phi}^{A}=$ 22094.67 and $\hat{\phi}^{B}=44366.67$. So $\hat{\phi}^{A}<\hat{\phi}^{B}$. Therefore our theoretical results state that species $B$ is the winner species. This is corroborated in the results of Figure 1. On the other hand, in brackish conditions, $\hat{\phi}^{A}=19834.67>17953.33=\hat{\phi}^{B}$ therefore species $A$ wins. In Figure 2, we see that this is indeed the case.

\section{Concluding Remarks}

We developed and analyzed a three-stage structured single population model and a two species model where each of the species is structured by three stages. For the single-species model, we 


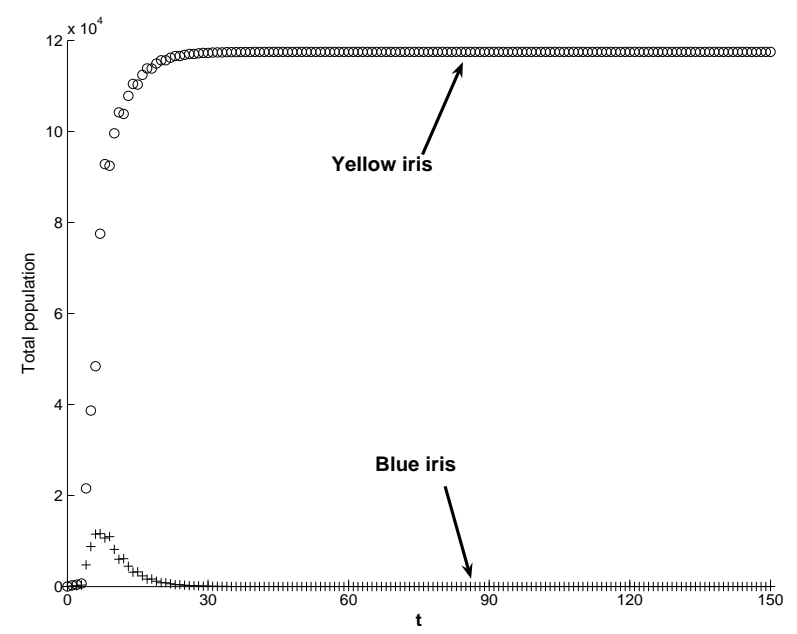

Figure 1: A plot of the total population for species $A$ and $B$ using parameter values $s_{1}^{A}=0.7, a_{2}^{A}=$ $0.8, a_{3}^{A}=0.9, b_{1}^{A}=117, b_{2}^{A}=1.08, s_{1}^{B}=0.6, a_{2}^{B}=0.8, a_{3}^{B}=0.9, b_{1}^{B}=276$ and $b_{2}^{B}=0.9$.

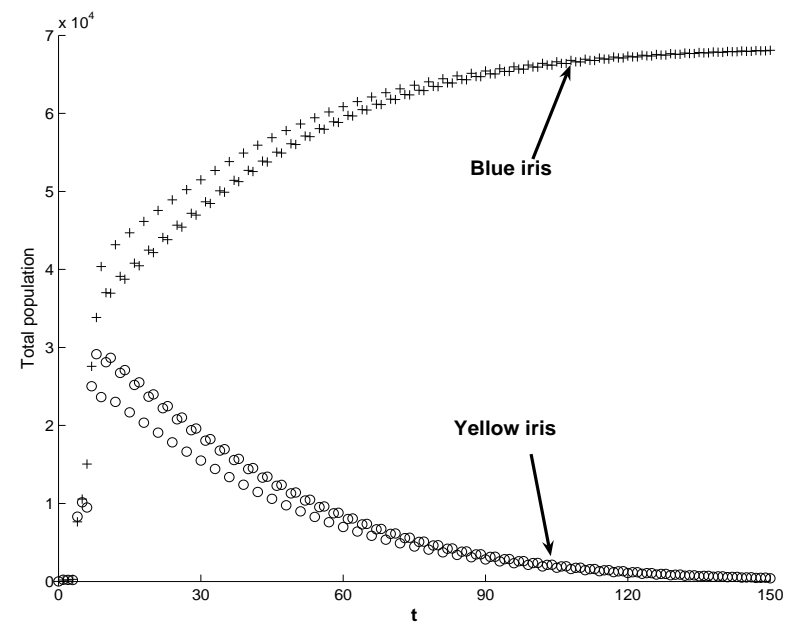

Figure 2: A plot of the total population for species $A$ and $B$ using parameter values $s_{1}^{A}=0.4, a_{2}^{A}=$ $0.8, a_{3}^{A}=0.6, b_{1}^{A}=186, b_{2}^{A}=0.48, s_{1}^{B}=0.4, a_{2}^{B}=0.6, a_{3}^{B}=0.5, b_{1}^{B}=226$ and $b_{2}^{B}=0.2$. 
showed that if the inherent net reproductive number, $R_{0}$, is less than one, then the model has only the trivial equilibrium, which results in population extinction; while if $R_{0}$ is greater than one, then the model has an interior equilibrium which is globally asymptotically stable.

Investigating the interaction between the two species we found that when both species have an inherent net reproductive number greater than one, the most likely outcome of competition between the two species is competitive exclusion where the winner species is the one which has higher density at which the net reproduction number is equal to one. We showed that coexistence equilibrium exists if $\hat{\phi}^{A}=\hat{\phi}^{B}$ with $R\left(\hat{\phi}^{A}\right)=1=R\left(\hat{\phi}^{B}\right)$. We also showed that under the simplified assumption that during one year all surviving seeds germinate and all surviving seedlings become flowering plants, competitive exclusion is the outcome for our model simulated with parameters chosen to resemble the blue and yellow irises in fresh and brackish water environments.

In this paper, we were interested in modeling competition between two species which have similar competition efficiencies. This, led to survivorship functions which are dependent on the total number of juveniles and adults of both species (denoted by $\phi$ ). The dynamics of the resulting model (3.1) are analogous to the classical continuous 2-dimensional Lotka-Volterra model (and to its discrete version studied in [6]) for the case when the nullclines do not intersect [2] . In particular, the resulting dynamics is competitive exclusion.

It is possible to obtain different dynamics including coexistence and bistability (founders effect) by allowing species to have different competition efficiencies. To this end, we modify the model (3.1) as follows:

$$
\left\{\begin{array}{l}
x_{t+1}^{A}=b_{1}^{A} z_{t}^{A} \\
y_{t+1}^{A}=s_{1}^{A} x_{t}^{A}+b_{2}^{A} z_{t}^{A} \\
z_{t+1}^{A}=s_{2}^{A}\left(\phi_{t}^{A}+c_{1} \phi_{t}^{B}\right) y_{t}^{A}+s_{3}^{A}\left(\phi_{t}^{A}+c_{1} \phi_{t}^{B}\right) z_{t}^{A} \\
x_{t+1}^{B}=b_{1}^{B} z_{t}^{B} \\
y_{t+1}^{B}=s_{1}^{B} x_{t}^{B}+b_{2}^{B} z_{t}^{B} \\
z_{t+1}^{B}=s_{2}^{B}\left(c_{2} \phi_{t}^{A}+\phi_{t}^{B}\right) y_{t}^{B}+s_{3}^{B}\left(c_{2} \phi_{t}^{A}+\phi_{t}^{B}\right) z_{t}^{B} .
\end{array}\right.
$$

Here $c_{1}$ and $c_{2}$ represent a measure of the strength of interspecific competition between the two species.

Using numerical simulations, we next show that indeed model (5.1) exhibits, in addition to competitive exclusion, coexistence and bistability. Therefore, the model (5.1) has similar dynamical behavior to the classical continuous 2-dimensional Lotka-Volterra model. In our numerical examples below, we will use $s_{2}^{A}\left(\phi_{1}\right)=a_{2}^{A} /\left(1+k_{1} \phi_{1}\right), s_{3}^{A}\left(\phi_{1}\right)=a_{3}^{A} /\left(1+k_{2} \phi_{1}\right), s_{2}^{B}\left(\phi_{2}\right)=$ $a_{2}^{B} /\left(1+k_{1} \phi_{2}\right)$, and $s_{3}^{B}\left(\phi_{2}\right)=a_{3}^{B} /\left(1+k_{2} \phi_{2}\right)$, where $\phi_{1}=\phi^{A}+c_{1} \phi^{B}$ and $\phi_{2}=c_{2} \phi^{A}+\phi^{B}$. The coefficients in the examples are $b_{1}^{A}=4, b_{1}^{B}=5, b_{2}^{A}=2, b_{2}^{B}=1, s_{1}^{A}=0.5, s_{1}^{B}=0.8, a_{2}^{A}=$ $0.6, a_{2}^{B}=0.7, a_{3}^{A}=0.4, a_{3}^{B}=0.6, k_{1}=0.01, k_{2}=0.1$.

In Figure 3, we let $c_{1}=0.4, c_{2}=0.2$, which means the interspecific competition levels are low. With initial conditions $\left(x_{0}^{A}, y_{0}^{A}, z_{0}^{A}\right)=(0,0,15)$ and $\left(x_{0}^{B}, y_{0}^{B}, z_{0}^{B}\right)=(0,0,10)$, the two species coexist. In Figure 4, we let $c_{1}=2, c_{2}=4$, which means the interspecific competition levels are high. Figure 4(a) shows that using the initial conditions $\left(x_{0}^{A}, y_{0}^{A}, z_{0}^{A}\right)=(0,0,15)$ and $\left(x_{0}^{B}, y_{0}^{B}, z_{0}^{B}\right)=(0,0,10)$ leads to the extinction of species B. Figure 4(b) shows that using the initial conditions $\left(x_{0}^{A}, y_{0}^{A}, z_{0}^{A}\right)=(0,0,10)$ and $\left(x_{0}^{B}, y_{0}^{B}, z_{0}^{B}\right)=(0,0,15)$ leads to the extinction of 
species A.

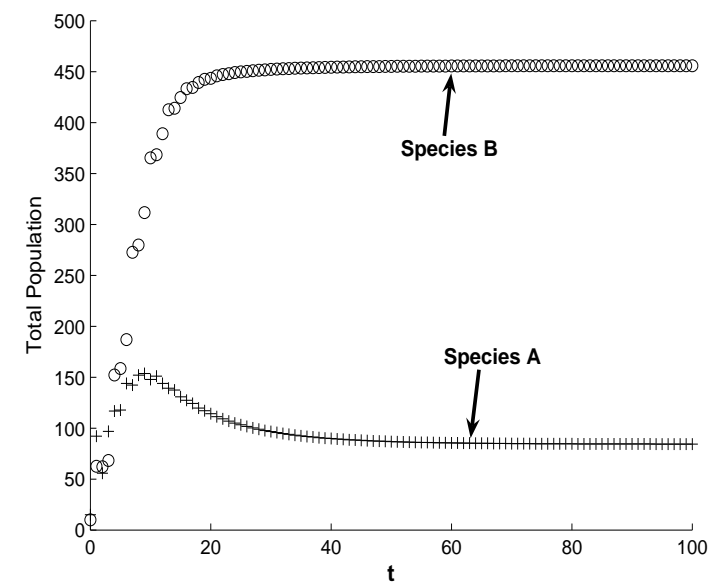

Figure 3: Coexistence of both species.

(a)

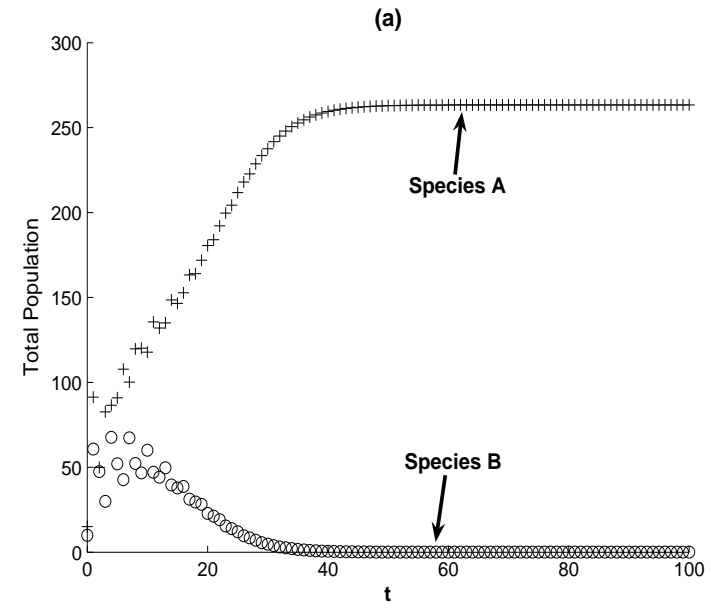

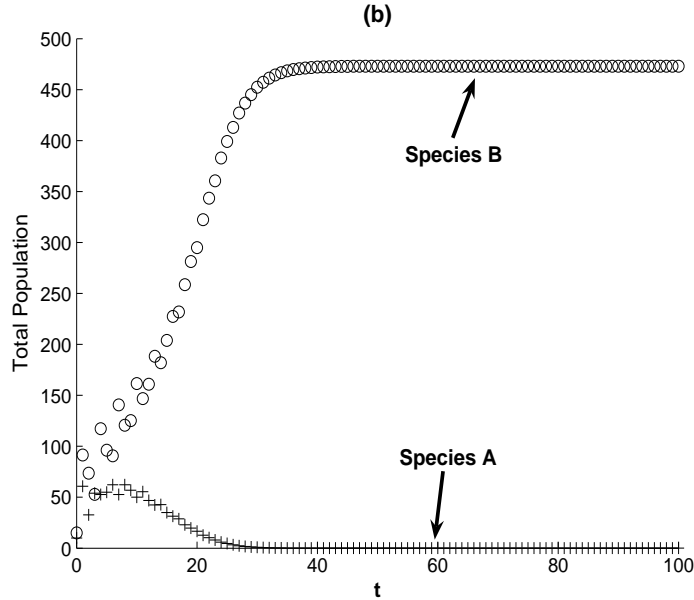

Figure 4: Bistablity (a) Species A persists (b) Species B persists.

In the future, we plan to theoretically investigate the dynamics of the more general model (5.1).

\section{Acknowledgement}

The work of A.S. Ackleh and P. Zhang is supported in part by the National Science Foundation under grant \# DUE-0531915. 


\section{References}

[1] A.S. Ackleh, P. DeLeenheer. Discrete three-stage population model: persistence and global stability results. J. Biol. Dyn., 2 (2008), 415-427.

[2] L.J.S. Allen. Introduction to mathematical biology. New Jersey. Prentice Hall, 2007.

[3] R.J.H. Beverton, S.J. Holt. On the dynamics of exploited fish populations. London. Fishery Investigations Series 2(19), Ministry of Agriculture, Fisheries and Food, 1957.

[4] H. Caswell. Matrix population models. 2nd Edition. Sunderland. Sinauer, 2001.

[5] J.M. Cushing. An introduction to structured population dynamics. Philadelphia. SIAM, 1998.

[6] J. M. Cushing, S. Levarge, N. Chitnis, S. M. Henson. Some discrete competition models and the competitive exclusion principle. J. Diff. Equa. Appl., 10 (2004), 1139-1151.

[7] J.M. Cushing, Z.Yicang. The net reproductive value and stability in matrix population models. Nat. Res. Mod., 8 (1994), 297-333.

[8] S. Elaydi. An introduction to difference equations. 3rd Edition. New York. Springer, 2005.

[9] S.B. Hsu, H.L. Smith, P. Waltman. Competitive exclusion and coexistence for competitive systems on ordered banach spaces. Trans. Amer. Math. Soci., 348(1996), 4083-4094.

[10] S. Pathikonda, A.S. Ackleh, K.H. Hasenstein, S. Mopper. Invasion, disturbance, and competition: modeling the fate of coastal plant populations. J. Cons. Biol., 23(2009), 164-173.

[11] W. Sutherland. Biological flora of the British Isles: Iris pseudacorus L. J. Eco., 78(1990), 833-848.

[12] W. Sutherland, D. Walton. The changes in morphology and demography of Iris pseudacorus L. at different heights on a saltmarsh. Func. Eco., 4(1990), 655-659.

[13] M. Tobler, P.V. Zandt, K. Hasenstein, S. Mopper. Above and belowground allocation in the wetland perennial, Iris hexagona: effects of population, salinity, and vertebrate florivory. Wetlands., 26(2006), 803-812.

[14] P. Viosca, Jr. The iries of southeastern Louisiana: a taxonomic and ecological interpretation. J. Amer. Iris. Soc., 57(1935), 3-56. 


\section{Appendix}

For convenience to the reader, we recall a few results that are utilized to prove global stability for the one and two species models. To this end we recall the following result [1] for monotone maps which is used to prove the global stability of the interior equilibrium for the one species model:

Lemma 2. Let $T: \mathbb{R}_{+}^{n} \rightarrow \mathbb{R}_{+}^{n}$ be a continuous, monotone map and $a \leq b$ be points in $\mathbb{R}_{+}^{n}$. If $a \leq T(a)$ and $T(b) \leq b$, and if $T$ has a unique fixed point $x^{*}$ in the order interval $[a, b]:=\{x \in$ $\left.\mathbb{R}_{+}^{n} \mid a \leq x \leq b\right\}$, then every solution sequence of the discrete system $x(t+1)=T(x(t))$, starting in $[a, b]$, converges to $x^{*}$.

Next we recall a result from [9] that we used to prove the global stability of the boundary equilibrium for the two species model. In what follows we use the exact notation used in [9]. For $i=1,2$, let $\mathbb{X}_{i}$ be ordered Banach spaces with positive cone $\mathbb{X}_{i}^{+}$such that Int $\mathbb{X}_{i} \neq \emptyset$. If $x_{i}, \bar{x}_{i} \in \mathbb{X}_{i}$, then we write $x_{i} \leq \bar{x}_{i}$ if $\bar{x}_{i}-x_{i} \in \mathbb{X}_{i}^{+}, x_{i}<\bar{x}_{i}$ if $x_{i} \leq \bar{x}_{i}$ and $x_{i} \neq \bar{x}_{i}$, and $x_{i} \ll \bar{x}_{i}$ if $\bar{x}_{i}-x_{i} \in$ Int $\mathbb{X}_{i}^{+}$. Let $\mathbb{X}=\mathbb{X}_{1} \times \mathbb{X}_{2}, \mathbb{X}^{+}=\mathbb{X}_{1}^{+} \times \mathbb{X}_{2}^{+}$, and $K=\mathbb{X}_{1}^{+} \times\left(-\mathbb{X}_{2}^{+}\right)$. $K$ has nonempty interior given by Int $K=\operatorname{Int} \mathbb{X}_{1}^{+} \times\left(-\operatorname{Int} \mathbb{X}_{1}^{+}\right)$. It generates the partial order relations $\leq_{K},<_{K}, \ll_{K}$. In this case, suppose that $x=\left(x_{1}, x_{2}\right), \bar{x}=\left(\bar{x}_{1}, \bar{x}_{2}\right) \in \mathbb{X}^{+}$, then

$$
\begin{aligned}
& x<_{K} \bar{x} \Longleftrightarrow x_{1}<\bar{x}_{1} \text { and } \bar{x}_{2}<x_{2} . \\
& x \ll_{K} \bar{x} \Longleftrightarrow x_{1} \ll \bar{x}_{1} \text { and } \bar{x}_{2} \ll x_{2} .
\end{aligned}
$$

Let $T: \mathbb{X}^{+} \rightarrow \mathbb{X}^{+}$be continuous and $T^{n}$ is the $n$-fold composition of $T$. Suppose that $x=\left(x_{1}, x_{2}\right)$ and $\bar{x}=\left(\bar{x}_{1}, \bar{x}_{2}\right) \in \mathbb{X}^{+}$. Consider the following hypotheses on $T$ :

(H1) $T$ is order compact and $x<_{K} \bar{x}$ implies $T(x)<_{K} T(\bar{x})$.

(H2) $T(0)=0$ and 0 is a repelling fixed point.

(H3) $T\left(\mathbb{X}_{1}^{+} \times\{0\}\right) \subset \mathbb{X}_{1}^{+} \times\{0\}$. There exists $\hat{x}_{1} \gg 0$ such that $T\left(\left(\hat{x}_{1}, 0\right)\right)=\left(\hat{x}_{1}, 0\right)$, and $T^{n}\left(\left(x_{1}, 0\right)\right) \rightarrow\left(\hat{x}_{1}, 0\right)$ for every $x_{1}>0$. The symmetric conditions hold for $T$ on $\{0\} \times \mathbb{X}_{2}^{+}$ with the fixed point denoted by $\left(0, \tilde{x}_{2}\right)$.

(H4) If $x, y \in \mathbb{X}^{+}$satisfy $x<_{K} y$ and either $x$ or $y$ belongs to Int $\mathbb{X}^{+}$, then $T(x) \ll_{K} T(y)$. If $x=\left(x_{1}, x_{2}\right) \in \mathbb{X}^{+}$satisfies $x_{i} \neq 0, i=1,2$, then $T(x) \gg 0$.

We denote the boundary fixed point of $T$ as: $E_{0}=(0,0), E_{1}=\left(\hat{x}_{1}, 0\right), E_{2}=\left(0, \tilde{x}_{2}\right)$ and the order interval $I:=\left[0, \hat{x}_{1}\right] \times\left[0, \tilde{x}_{2}\right]$. Then we have the following theorem[9]:

Theorem 4. Let (H1)-(H4) hold. Then the omega limit set of every orbit is contained in I and exactly one of the following holds:

(a) There exists a positive fixed point $E^{*}$ of $T$ in $I$.

(b) $T^{n}(x) \rightarrow E_{1}$ as $n \rightarrow \infty$ for every $x=\left(x_{1}, x_{2}\right) \in I$ with $x_{i} \neq 0, i=1,2$. 
(c) $T^{n}(x) \rightarrow E_{2}$ as $n \rightarrow \infty$ for every $x=\left(x_{1}, x_{2}\right) \in I$ with $x_{i} \neq 0, i=1,2$.

Finally, if $(b)$ or (c) holds and $x=\left(x_{1}, x_{2}\right) \in X^{+} \backslash I$ satisfies $x_{i} \neq 0, i=1,2$, then either $T^{n}(x) \rightarrow E_{1}$ as $n \rightarrow \infty$ or $T^{n}(x) \rightarrow E_{2}$ as $n \rightarrow \infty$.

As stated in [9] Theorem 4 is still true if (H4) is replaced by the following (H4)'.

(H4)' If $x, y \in \mathbb{X}^{+}$satisfy $x<_{K} y$ and either $x$ or $y$ belongs to Int $\mathbb{X}^{+}$, then $T^{l}(x) \ll_{K} T^{l}(y)$ for some $l \in \mathbb{Z}^{+}$; If $x=\left(x_{1}, x_{2}\right) \in \mathbb{X}_{1}^{+} \times \mathbb{X}_{2}^{+}$satisfies $x_{i} \neq 0, i=1,2$, then $T^{m}(x) \gg 0$, for some $m \in \mathbb{Z}^{+}$. 